\title{
Does Neurofeedback Training Improve Performance in
} Athletes?

\author{
Christophe Domingos ${ }^{1,2^{*}}$, Cristiana P. Alves ${ }^{2}$, Edgar Sousa $^{1}$, Agostinho Rosa $^{2}$, and José G. \\ Pereira ${ }^{1}$ \\ ${ }^{1}$ Laboratory of Physiology and Biochemistry of Exercise, Faculty of Human Kinetics, University of Lisbon, Cruz \\ Quebrada, Portugal \\ 2Department of Bioengineering, LaSEEB - System and Robotics Institute, Instituto Superior Técnico, University of \\ Lisbon, Lisbon, Portugal
}

\begin{tabular}{|c|c|}
\hline \multicolumn{2}{|c|}{ Abstract } \\
\hline \multicolumn{2}{|c|}{$\begin{array}{l}\text { Introduction: Neurofeedback training has been an increasingly used technique in sport; however, most of the } \\
\text { protocols used in athletes are based in the results obtained in nonathletic population. Purpose: Understand if a } \\
\text { specific neurofeedback training protocol implemented in a nonathletic population can improve short-term memory } \\
\text { and reaction time in athletes. Methods: A total of } 45 \text { subjects participated in the experiment (mean } \pm S D \text { for age: } \\
23.31 \pm 4.20 \text { years). For athletes, } 12 \text { neurofeedback training sessions were performed; for the nonathletes, } 15 \\
\text { neurofeedback training were performed. Each session had } 25 \text { min of effective neurofeedback training. Results: } \\
\text { Despite the nonathletes group's increased standard alpha band (SAB) relative amplitude and individual alpha } \\
\text { band (IAB) relative amplitude after } 12 \text { sessions of neurofeedback training }(p<.005) \text {, only the athletes intervention } \\
\text { group had positive results in reaction time ( } p<.001 \text { in oddball test). Not only was the null hypothesis rejected } \\
\text { by the differences of IAB and SAB relative amplitudes between and within protocols but also by the performance } \\
\text { tests. Conclusion: Neurofeedback training increases the relative amplitude of the bands in the nonathletes } \\
\text { group; however, only the athletes have shown to improve performances tests after } 12 \text { neurofeedback training } \\
\text { sessions. }\end{array}$} \\
\hline \multicolumn{2}{|c|}{$\begin{array}{l}\text { Keywords: neurofeedback training; athletic training; reaction time; short-term memory; performance; individual } \\
\text { alpha band }\end{array}$} \\
\hline \multicolumn{2}{|c|}{$\begin{array}{l}\text { Citation: Domingos, C., Alves, C. P., Sousa, E., Rosa, A., \& Pereira, J. G. (2020). Does neurofeedback training improve performance in } \\
\text { athletes? NeuroRegulation, 7(1), 8-17. https://doi.org/10.15540/nr.7.1.8 }\end{array}$} \\
\hline $\begin{array}{l}\text { ^Address correspondence to: Christophe Domingos, Faculdade de } \\
\text { Motricidade Humana (FMH), Universidade de Lisboa (UL), Estrada } \\
\text { da Costa MB, 1499-002, Cruz Quebrada - Dafundo, Portugal. Email: } \\
\text { christophedomingos@campus.ul.pt }\end{array}$ & $\begin{array}{l}\text { Edited by: } \\
\text { Rex L. Cannon, PhD, SPE } \\
\text { Tennessee, USA }\end{array}$ \\
\hline $\begin{array}{l}\text { Copyright: }(2020 \text {. Domingos et al. This is an Open Access article } \\
\text { distributed under the terms of the Creative Commons Attribution } \\
\text { License (CC-BY). }\end{array}$ & $\begin{array}{l}\text { Reviewed by: } \\
\text { Rex L. Cannon, PhD, SPESA Research Institute, Knoxville, } \\
\text { Tennessee, USA } \\
\text { Randall Lyle, PhD, Mount Mercy University, Cedar Rapids, lowa, } \\
\text { USA }\end{array}$ \\
\hline
\end{tabular}

\section{Introduction}

Neurofeedback has gained interest in professional sports and performance in order to better understand the neural influences on sporting behaviors and to improve performance (Park, Fairweather, \& Donaldson, 2015). Elite athletes provide an exemplary model for understanding the effects of mastery, expertise, and execution of such skills (Park et al., 2015). In order to achieve the elite level, in almost all sports, athletes take two to two and a half decades of apprenticeship involving self-control, skill learning, long-term planning, and resilience to failure, judgment, defeat, and injury (Walsh, 2014). Neurofeedback training (NFT) has produced robust findings in health (Marzbani, Marateb, \& Mansourian, 2016; Noakes, 2012), and cognition and performance (Cross, Acquah, \& Ramsey, 2014; Vernon, 2005). NFT in sports typically involves application of traditional protocols used in the general population or in mental health treatment. To date, few protocols have been developed for professional athletes to improve performance or identify specific neural targets associated with enhancement of performance. 
Electroencephalography (EEG) is a procedure used to record the electrical activity of the brain on the scalp. Neurofeedback provides the individual feedback about this activity to potentially enhance performance in sport by retraining this activity (Mirifar, Beckmann, \& Ehrlenspiel, 2017). Data have revealed distinct cortical differences between expert athletes and nonexperts (Landers et al., 1991; Salazar et al., 1990; Vernon, 2005). These results are in line with the neural efficiency hypothesis (Babiloni et al., 2010) that is based on the specific activation brain regions for a given task while disengaging irrelevant brain regions for the same task (Haier, Siegel, Tang, Abel, \& Buchsbaum, 1992). It is a phenomenon that can easily be found in sport and even more in elite athletes (Milton, Solodkin, Hluštík, \& Small, 2007). Data have shown that elite karate athletes show a less pronounced alpha event-related desynchronization (ERD) than amateur karate athletes during performance, and this reinforces the widely held idea that elite athletes utilize specialized zones for a particular action (Babiloni et al., 2010). This is suggested to be related to the existence of several frequency bands and the mental state that are associated to each frequency (Thompson \& Thompson, 2015); that is, the alpha band relates to a state of relaxed attention (Klimesch, 1999), processing speed (Angelakis et al., 2007), better memory function (Guez et al., 2015; Nan et al., 2012), and reaction time (Ziółkowski et al., 2012), for example. It is hypothesized that those differences are not consistent in location nor direction, which might be explained by different sport-specific requirements (Vernon, 2005) or, in the case of the alpha band, by the several intervals suggested (da Silva, 2013; Thompson \& Thompson, 2015). Mirifar, Beckmann, and Ehrlenspiel (2017) rightly propose the need to understand the cortical activity (site)-personalized event-locked EEG profile-that is associated with performance (Mirifar et al., 2017), which is still unrealistic due to the impossibility to assess in sports involving head movement (creating artifacts). However, it is possible to work at the individual alpha band (IAB) to specialize NFT, reducing variability in training (Bazanova \& Mernaya, 2008; Klimesch, 1999). As Mirifar et al. (2017) point out in a recent systematic review, many protocols applied in sport are based on positive results found outside the area of interest (Mirifar et al., 2017).

The primary aim of this study was to evaluate the effects of an NFT protocol on short-term memory and reaction time in high performance athletes as compared to nonathletes. To understand the effects of NFT, a group of athletes (intervention group) who performed NFT sessions was compared with another group of athletes (control group) who did not perform any NFT sessions. A nonathlete group (intervention group) was also added to the study, with a similar protocol, to comprehend if the protocols should be adapted according to the populations studied. To the best of our knowledge, no study compared the effects of NFT between two athletic populations and between a nonathletic population at the same time, with the same protocol under the same conditions. We hypothesize that (a) the standard alpha band (SAB) relative amplitude and the $I A B$ relative amplitude in NFT sessions are similar between intervention groups (similar margin progression) and (b) the performance tests (memory and reaction time) results will be the same after all NFT sessions in both groups (intervention groups and control group).

\section{Methods}

\section{Subjects}

A total of 45 subjects aged from 18 to 44 years old participated in the experiment (mean $\pm S D$ for age: $23.31 \pm 4.20$ years). All student athletes have been involved in federated sports or practicing exercise or sport regularly for more than 5 years (Baker, Côté, \& Deakin, 2005), as compared to the group of nonathlete students that do not meet the minimum five times a week of at least moderate intensity requirements to be considered active (World Health Organization, 2010). The inclusion criteria were as follows:

- no history of psychiatric or neurological disorders;

- no psychotropic medications or addiction drugs;

- normal or corrected-to-normal vision;

- minimum age of 18 years and maximum age of 45 years; and

- $\quad$ ractice moderate-intensity exercise at least 5 times a week (sport or gym) regardless of skill level (for athlete groups).

\begin{tabular}{lccc}
\hline \multicolumn{3}{l}{ Table 1} \\
\multicolumn{4}{l}{ Age for each group, } \\
\hline & $\begin{array}{c}\text { Control } \\
(n=15)\end{array}$ & $\begin{array}{c}\text { Athletes } \\
(n=15)\end{array}$ & $\begin{array}{c}\text { Nonathlete } \\
(n=15)\end{array}$ \\
\hline $\begin{array}{c}\text { Age } \\
\text { (years) }\end{array}$ & $22.53 \pm 3.89$ & $27.93 \pm 6.11$ & $21.20 \pm 2.62$ \\
\hline
\end{tabular}

All students were informed about the possible risks of the investigation before providing written informed consent to participate. All procedures were approved 
by the Ethics Committee of the Faculty of Human Kinetics and Instituto Superior Técnico, University of Lisbon, and conducted in accordance with the Declaration of Helsinki (World Medical Association, 2001). All data collected has been stored in a database where only researchers related to the NFT project have access. Anonymity was guaranteed.

\section{Signal Acquisition}

After being carefully informed on capping, signal collection, and inherent processes of artifact production, participants sat in a room with a controlled environment. The EEG signals were recorded according to the international 10-20 system (Fp1, Fp2, F3, F4, F7, F8, C3, C4, T3, T4, P3, P4, T5, T6, $\mathrm{O} 1, \mathrm{O} 2, \mathrm{Fz}, \mathrm{Cz}$, and $\mathrm{Pz}$ ), with a sampling frequency of $256 \mathrm{~Hz}$. Feedback was from $\mathrm{Cz}$ channel (it was chosen since it is at the primary motor cortex and has been associated with sensory information processing over the sensorimotor area and provide a measurement of the activity in both hemispheres and in the frontal lobe; Mann, Sterman, \& Kaiser, 1996; Pfurtscheller, Neuper, Ramoser, \& Müller-Gerking, 1999). The ground was located at forehead and the reference was the average of left and right mastoids. The signals were amplified by a 24-channel system (Vertex 823 from Meditron Electomedicina Ltda, SP, Brazil) and were recorded by Somnium software platform (Cognitron, SP, Brazil) and NF module by Laseeb-ISR. Circuit impedance was kept below 10 $\mathrm{k} \Omega$ for all electrodes before the sessions. Subjects were asked to sit comfortably and then to remain as still as possible and also to avoid excessive blinking and abrupt movements.

\section{Experimental Design}

In the first session of this randomized controlled study, all intervention participants performed a 5-min NFT familiarization to understand how to achieve alpha band mental state (increase the alpha amplitude [i.e., power] and the time remaining within this range; Bazanova \& Vernon, 2014) or, in other words, to understand how to remain in a concentration state by the real feedback presented on the screen (Thompson \& Thompson, 2015), followed by the pretests (the performance tests are the same and will be described in the Assessments section). The pre- and posttests had the same interval of time for both the control and intervention groups. Timeline of the NFT training sessions and respective performance tests (pre- and posttests) are presented in Figure 1.

Intervention group - Athletes. The intervention group performed a familiarization session and pretest before $\left(\mathrm{bS}^{1}\right)$ the 12 NFT sessions. Between session 6 and session $7\left(S^{6 / 7}\right)$, performance tests were applied. At the end, a posttest $\left(\mathrm{aS}^{12}\right)$ was performed. The NFT sessions consisted in 25 trials of $60 \mathrm{~s}$ each with $5 \mathrm{~s}$ of pause between trials. The total NFT session time for each subject was $300 \mathrm{~min}$. The NFT sessions were performed two times per week. Although inhibiting self-talk seems to be one of the best strategies (Harkness, 2009; Hatfield, Haufler, \& Spalding, 2006; Hosseini \& Norouzi, 2017; Kamata, Tenenbaum, \& Hanin, 2002; Wilson, Peper, \& Moss, 2006), participants were only requested to concentrate on their sport activity as much as possible but not in a specific task.

Intervention group - Nonathletes. The intervention group performed a familiarization session and pretest before $\left(\mathrm{bS}^{1}\right)$ the 15 NFT sessions. Between session 5 and session $6\left(S^{5 / 6}\right)$, performance tests were applied. Also, between session 10 and session 11 $\left(S^{10 / 11}\right)$, performance tests were applied. At the end, a posttest $\left(\mathrm{aS}^{15}\right)$ was performed. The NFT involved five blocks of trials. Each block was constituted by five 1-min trials with $5 \mathrm{~s}$ between trials. In this way each session had 25 min of effective NFT, and each protocol resulted in $375 \mathrm{~min}$.

Control group. The control group only performed pre- $\left(\mathrm{bS}^{1}\right)$ and posttests $\left(\mathrm{aS}^{12}\right)$ over a month and a half without the training sessions. 


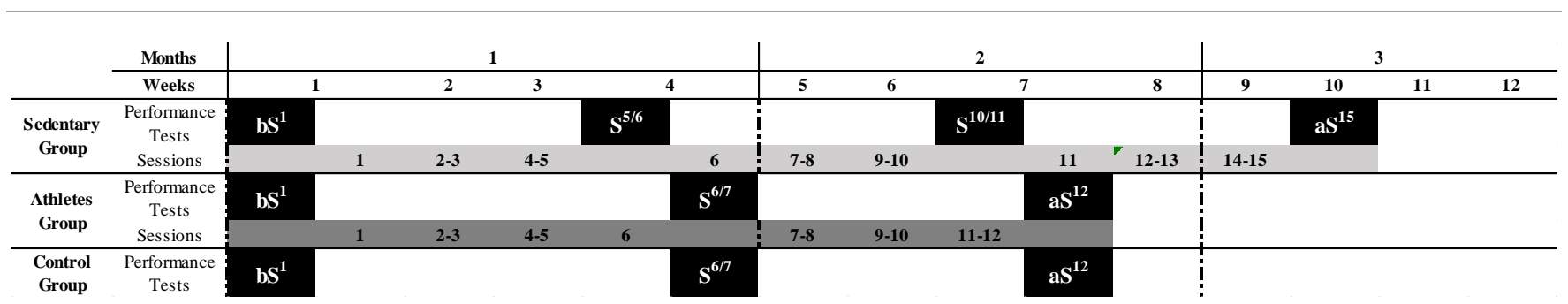

Figure 1. Timeline of the NFT training sessions and respective performance tests $\left(b S^{1}, S^{5 / 6}, S^{10 / 11}\right.$, and aS $S^{15}$ for nonathletes group; and $\mathrm{bS}^{1}, \mathrm{~S}^{5 / 6}$, and $\mathrm{aS}^{12}$ for athletes group and control group).

\section{Measurements}

The baseline individual alpha frequency (IAF) was determined before and after NFT. The baseline recording consisted of 2 min during the resting period, altering between eyes closed and eyes open. Recordings of eyes open and closed in baseline 1 provided data for the calculation of alpha desynchronization and synchronization respectively; this enabled determination of individual frequency bands through amplitude band crossings (Klimesch, 1999).

Feedback is a determinant step for the protocol's success. Neural activity must be fed back by some parameter(s) and presented to the participant in a simple and direct representation of their value. In this study, the feedback parameter was the relative amplitude of the IAB calculated as in Equation 1 where band amplitude was the amplitude of the IAB and EEG amplitude was the amplitude from $4 \mathrm{~Hz}$ to $30 \mathrm{~Hz}$. Using the amplitude spectrum instead of the power spectrum prevents excessive skewing which results from squaring the amplitude, and thus increases statistical validity.

Relative Amplitude $=\frac{\text { Band Amplitude }}{\text { EEG Amplitude }}$

The visual feedback display contains two tridimensional objects: a sphere and a cube. The sphere radius reflects the feedback parameter value in real time and if it reaches a threshold (Goal 1) its colour changes. The sphere has several slices (initially four, the minimum), and the more present, the smoother it looks. While Goal 1 is being achieved, slices are added; if not, the sphere loses them until it has four again. The cube height is related to the period of time that Goal 1 kept being achieved continuously. If it happens for more than a predefined period of time (2 s), Goal 2 is accomplished, and the cube rises until Goal 1 stops being achieved. Then it starts falling until it reaches the bottom or Goal 2 is achieved again. Therefore, the participant's task is to take the cube as high as possible (Rodrigues, Migotina, \& da Rosa, 2010).

The feedback threshold was set to 1.0 in the first session, and it was adjusted according to the session report which showed the percentage of time for which the feedback parameter was above the threshold in each session. If this percentage exceeded $60 \%$, the threshold would be increased by 0.1 in the next session. In contrast, if the percentage was below $20 \%$, the threshold would be decreased by 0.1 in the next session (Nan, Wan, Lou, Vai, \& Rosa, 2013).

\section{Assessments}

Digit Span (DS). Participants had to recall a random sequence of numbers in the correct order, starting with 2 digits and ending with 10 digits. Subjects were asked to introduce the digits in the order by which they appeared (YuLeung To, Abbott, Foster, \& Helmer, 2016).

Oddball (OB). The oddball test is used to evaluate the attention of the subjects. In this test, different geometrical forms appear (circle, octagon, and square) and the participants were instructed to click only if the circle appeared. The test consisted of 50 trials, where the images appeared during $0.5 \mathrm{~s}$ with an interval of $0.5 \mathrm{~s}$. It was defined by a decoy rate of 40\% (Debener, Makeig, Delorme, \& Engel, 2005).

\section{Statistical Analysis}

Differences in $\mathrm{SAB}$ and $\mathrm{IAB}$ bands and performance tests over time for all groups were examined using the ANOVA test and a post hoc Friedman test was performed when normality was not verified and to evaluate significant differences between groups. Comparison of performance tests means and differences between groups were performed using the ANOVA test and the post hoc Kruskal-Wallis was performed when normality was not verified and to know between which groups there were significant differences, a post hoc Tukey's test was performed. Data were analyzed with SPSS software for Windows 
version 25.0 (SPSS Inc., Chicago, IL). Statistical significance was set at $p<.05$ for all tests.

\section{Results}

Although both populations increase $S A B$ and $I A B$ throughout the sessions, when comparing the groups there are no differences.

The $S A B$ and $I A B$ over the 12 sessions in both protocols during NFT sessions are presented in
Figure 2. Both populations show positive slopes, but in the nonathlete population it is clearly verified that there is an effect of the sessions in the increase of $\mathrm{SAB}\left(R^{2}=.864\right)$ and IAB $\left(R^{2}=.904\right)$. Only in the nonathlete population are there significant results in both $\mathrm{SAB}$ between session 1 and 12 (1.01 \pm 0.13 vs. $1.15 \pm 0.22 ; p=.018)$ and $I A B$ between sessions 1 and $10(1.04 \pm 0.17$ vs. $1.19 \pm 0.21 ; p=.003)$ and sessions 1 and $12(1.04 \pm 0.17$ vs. $1.19 \pm 0.22 ; p$ $=.003)$. In other words, $S A B$ and IAB significantly increase from session 1 to $12(p<.05)$.
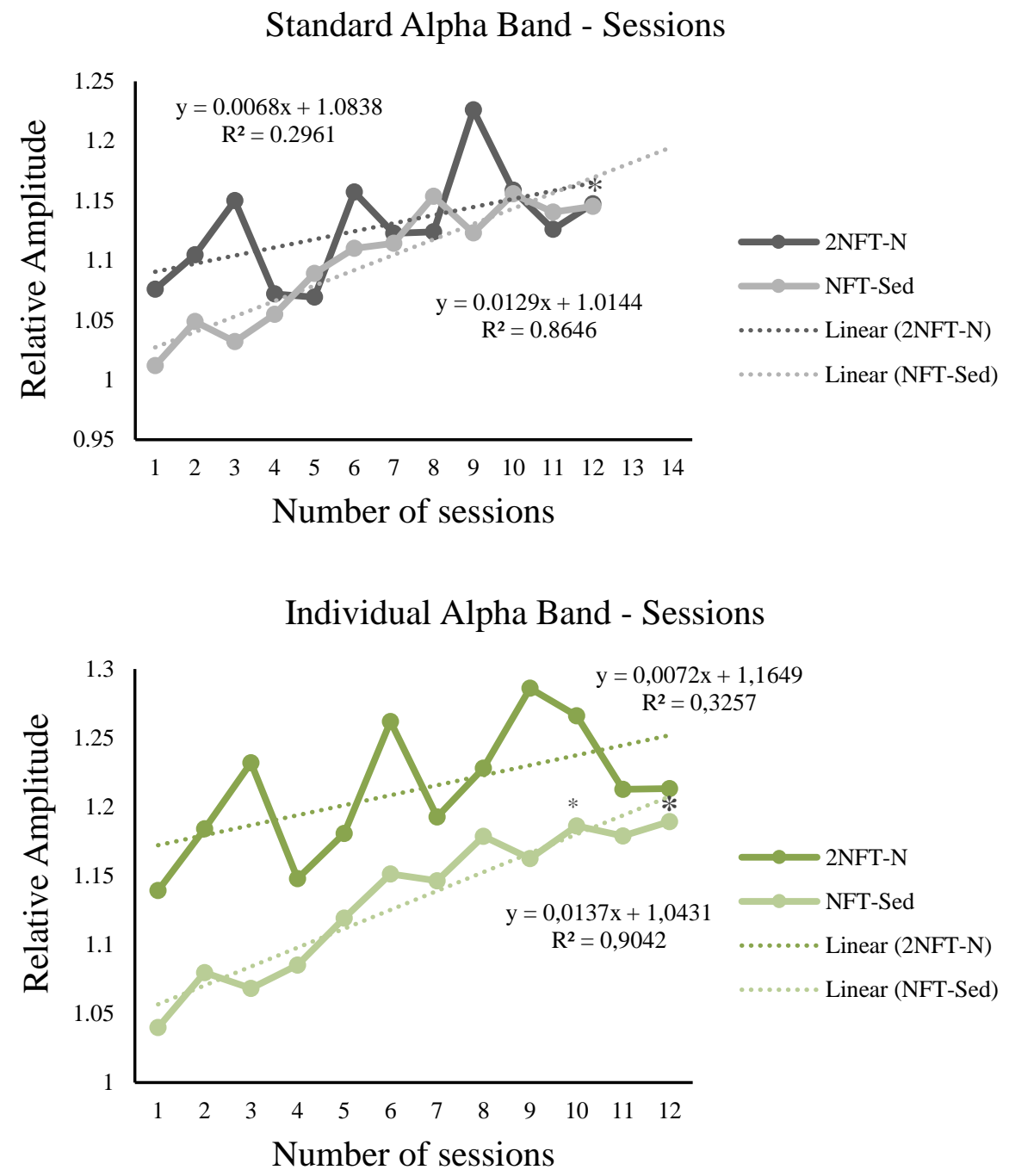

Figure 2. Differences between session 1 and 12 and in standard alpha band (SAB; top image) and individual alpha band (IAB; bottom image) for each protocol ${ }^{*}(p<.05)$.

Differences in performance tests between both populations and control group are presented in Table 2. Only differences were found between the nonathlete group and the athletes group in DS $S^{a / b}$ tests. The OB bS ${ }^{1}$ tests, OB Sa/b tests, OB Sc/d tests, and $O B S^{d / e}$ tests showed differences between the nonathlete population and the athletes population and control group. 


\section{Table 2}

Differences in performance tests $\left(b S^{1}, S^{5 / 6}, S^{10 / 11}\right.$, and $a S^{15}$ for nonathlete group; and $b S^{1}, S^{5 / 6}$, and $a S^{12}$ for athletes group and control group) between protocols, $M \pm S D$.

\begin{tabular}{|c|c|c|c|c|}
\hline & $\begin{array}{l}\text { Control }\left.\right|^{\mathrm{b}, \mathrm{d}} \\
(n=15)\end{array}$ & $\begin{array}{l}\text { Athletes }{ }^{\mathrm{b}, \mathrm{d}} \\
(n=15)\end{array}$ & $\begin{array}{l}\text { Nonathlete } \mathrm{e}^{\mathrm{a}, \mathrm{c}, \mathrm{e}} \\
\quad(n=15)\end{array}$ & $p$ \\
\hline $\mathrm{DS}_{\mathrm{bS}}{ }^{1}$ tests & $7.13 \pm 1.36$ & $7.20 \pm 0.94$ & $6.50 \pm 1.65$ & $.377^{f}$ \\
\hline$D S S^{a / b}$ tests & $7.53 \pm 1.36$ & $7.87 \pm 0.74$ & $6.36 \pm 1.45$ & $.003^{f}$ \\
\hline DS Sc/d tests & $7.93 \pm 0.96$ & $8.13 \pm 0.83$ & $7.07 \pm 1.73$ & $.064^{f}$ \\
\hline DS S $\mathrm{d} / \mathrm{e}$ tests & $7.93 \pm 0.96$ & $8.13 \pm 0.83$ & $7.77 \pm 1.92$ & $.575^{f}$ \\
\hline Difference in DS ( $\mathrm{S}^{\mathrm{a} / \mathrm{b}}-\mathrm{S}^{1}$ tests) & $0.40 \pm 1.24$ & $0.67 \pm 0.98$ & $-0.14 \pm 1.03$ & $.137^{f}$ \\
\hline Difference in DS $\left(S^{c / d}-S^{a / b}\right)$ & $0.40 \pm 0.91$ & $0.27 \pm 0.88$ & $0.71 \pm 1.07$ & $.578^{f}$ \\
\hline Difference in DS $\left(S^{c / d}-S^{1}\right)$ & $0.80 \pm 1.08$ & $0.93 \pm 1.22$ & $0.57 \pm 1.55$ & $.913^{f}$ \\
\hline Difference in DS (Sd/e $\left.-S^{a / b}\right)$ & $0.40 \pm 0.91$ & $0.27 \pm 0.88$ & $1.31 \pm 1.38$ & $.070^{f}$ \\
\hline Difference in DS ( $\left.S^{d / e}-S^{1}\right)$ & $0.80 \pm 1.08$ & $0.93 \pm 1.22$ & $1.15 \pm 1.77$ & $.953^{f}$ \\
\hline OB $S^{1}$ tests & $94.80 \pm 5.28$ & $95.20 \pm 3.84$ & $83.29 \pm 8.83$ & $<.001^{f}$ \\
\hline$O B S^{a / b}$ tests & $96.27 \pm 3.45$ & $98.00 \pm 2.00$ & $88.29 \pm 5.06$ & $<.001^{f}$ \\
\hline OB Sc/d tests & $96.27 \pm 3.01$ & $98.53 \pm 1.41$ & $82.43 \pm 16.04$ & $<.001^{f}$ \\
\hline OB S $\mathrm{d} / \mathrm{e}$ tests & $96.27 \pm 3.01$ & $98.53 \pm 1.41$ & $86.00 \pm 8.29$ & $<.001^{f}$ \\
\hline Difference in OB ( $\mathrm{S}^{\mathrm{a} / \mathrm{b}}-\mathrm{S}^{1}$ tests) & $1.47 \pm 3.81$ & $2.80 \pm 3.10$ & $5.00 \pm 8.07$ & $.175^{\dagger}$ \\
\hline Difference in OB $\left(\mathrm{S}^{\mathrm{c} / \mathrm{d}}-\mathrm{S}^{\mathrm{a} / \mathrm{b}}\right)$ & $0.00 \pm 3.21$ & $0.53 \pm 1.41$ & $-5.86 \pm 14.43$ & $.503^{f}$ \\
\hline Difference in OB $\left(S^{c / d}-S^{1}\right)$ & $1.47 \pm 2.77$ & $3.33 \pm 3.44$ & $-0.86 \pm 13.24$ & $.421^{f}$ \\
\hline Difference in OB $\left(S^{d / e}-S^{a / b}\right)$ & $0.00 \pm 3.21$ & $0.53 \pm 1.41$ & $-1.85 \pm 6.80$ & $.767^{\dagger}$ \\
\hline Difference in $\mathrm{OB}\left(\mathrm{S}^{\mathrm{d} / \mathrm{e}}-\mathrm{S}^{1}\right)$ & $1.47 \pm 2.77$ & $3.33 \pm 3.44$ & $3.69 \pm 9.34$ & $.360^{f}$ \\
\hline \multicolumn{5}{|c|}{ 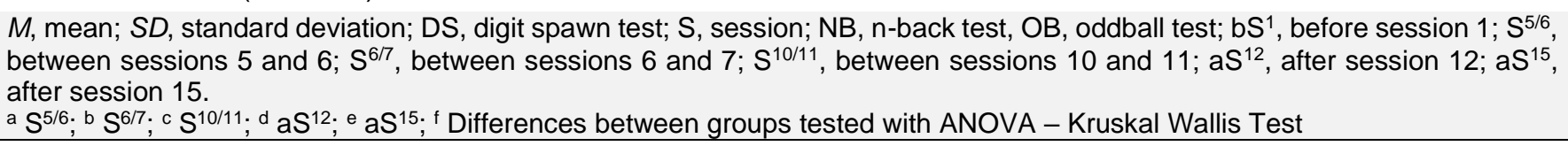 } \\
\hline
\end{tabular}

Table 3 shows the differences between performance tests for athletes, nonathletes, and control group. In the control group differences were found for the DS, where participants improved the score between bS ${ }^{1}$ tests and $S^{c / d}$ tests. In the athlete population, results were found in both DS and OB between $\mathrm{bS}^{1}$ tests and $\mathrm{S}^{\mathrm{c} / \mathrm{d}}$ tests. In the nonathlete population differences were only found from $S^{a / b}$ tests to $S^{e}$ tests. 


\begin{tabular}{|c|c|c|c|c|c|}
\hline \multicolumn{6}{|c|}{$\begin{array}{l}\text { Differences between } b S^{1}, S^{5 / 6}, S^{10 / 11} \text {, and } a S^{15} \text { for nonathlete group and } b S^{1}, S^{5 / 6} \text {, and } a S^{12} \text { for athletes group } \\
\text { and control group for each protocol, } M \pm S D \text {. }\end{array}$} \\
\hline & $\mathrm{bS}^{1}$ tests & $\mathrm{S}^{\mathrm{a} / \mathrm{b}}$ tests & $\mathrm{S}^{\mathrm{c} / \mathrm{d}}$ tests & $\mathrm{S}^{\mathrm{d} / \mathrm{e}}$ tests & $p$ \\
\hline \multicolumn{6}{|l|}{ Control } \\
\hline DS & $7.13 \pm 1.36$ & $7.53 \pm 1.36$ & $7.93 \pm 0.96$ & $\mathrm{~N} / \mathrm{A}$ & $.031^{g}$ \\
\hline $\mathrm{OB}$ & $94.80 \pm 5.28$ & $96.27 \pm 3.45$ & $96.27 \pm 3.01$ & $\mathrm{~N} / \mathrm{A}$ & $.161^{g}$ \\
\hline \multicolumn{6}{|l|}{ Athletes ${ }^{b, d}$} \\
\hline DS & $7.20 \pm 0.94$ & $7.87 \pm 0.74$ & $8.13 \pm 0.83$ & $\mathrm{~N} / \mathrm{A}$ & $.022^{g}$ \\
\hline $\mathrm{OB}$ & $95.20 \pm 3.84$ & $98.00 \pm 2.00$ & $98.53 \pm 1.41$ & $\mathrm{~N} / \mathrm{A}$ & $<.0019$ \\
\hline \multicolumn{6}{|c|}{ Nonathletes a, c, e } \\
\hline DS & $6.50 \pm 1.65$ & $6.36 \pm 1.45$ & $7.07 \pm 1.73$ & $7.77 \pm 1.92$ & $.006^{f}$ \\
\hline $\mathrm{OB}$ & $83.29 \pm 8.83$ & $88.29 \pm 5.06$ & $82.43 \pm 16.04$ & $86.00 \pm 8.29$ & $.171 \mathrm{~g}$ \\
\hline \multicolumn{6}{|c|}{ 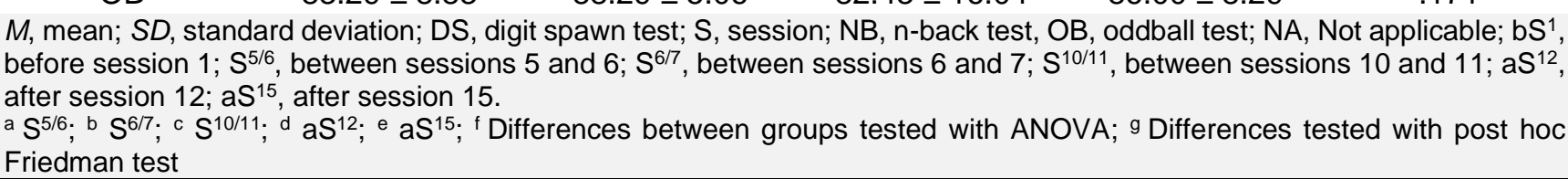 } \\
\hline
\end{tabular}

The primary aim of the study was to analyze if the number of NFT sessions would be enough to increase the relative amplitude of $S A B$ and $I A B$ in both intervention groups. Knowing the results of relative amplitude changes, a secondary aim was to understand a possible link between NFT sessions $S A B$ and $I A B$ in both intervention groups and control group and the performance tests.

Our results demonstrated that only the nonathlete group increased $S A B$ and $I A B$ after 12 sessions of NFT. However, only the athletes intervention group had positive results in reaction time (i.e., in OB test). This finding lends preliminary support to the alternative hypothesis that the $S A B$ and IAB will be different between groups, which means that the nonathlete population present a different progression margin from the athlete population. It is also important to note that the nonathlete group only had results in short-memory performance test after 15 sessions, while the athlete group only required 12 sessions, and the control group did not require NFT sessions to have results in this test. Not only was the null hypothesis rejected by the differences of IAB and $S A B$ between and within protocols but also by the performance tests.

\section{Discussion}

The results that should be considered the key point of the study relate to the reaction time performance test. They suggest that the number of sessions in athletes are sufficient to have positive changes when compared to the nonathlete group that did not improve positively in OB performance test and when compared to the control group who also did not improve in this test.

\section{SAB and IAB Over Time Results}

As it can be seen in Figure 2, both intervention groups had a positive slope in $S A B$ and IAB bands which demonstrates the effects of NFT over time. However, the $S A B$ and IAB slopes in the nonathlete group were steeper, due to the lower initial value when compared to the athletes. Once again, it is demonstrated that athletes have a lower progression margin $\left(R^{2}=0.32\right)$ than the nonathlete group $\left(R^{2}=0.90\right)$ because they have higher initial values. Another curious fact that can be verified in Figure 2 is the behaviour of the bands throughout the sessions; that is, the group of athletes presents a more cyclic behaviour while the nonathlete group presents a more linear evolution. Yet, the discrete variation seen in the session intervals for SAB (Maszczyk et al., 2018; Thompson, Steffert, Ros, Leach, \& Gruzelier, 2008) and for IAB (Egner \& Gruzelier, 2001; Maszczyk et al., 2018) suggest different behavioral patterns as a function of number of sessions. The baseline values in $\mathrm{SAB}$ and $I A B$ for both groups are slightly different but sufficient so that the interval from session 1 to session 12 demonstrates differences only in the nonathlete group. It can be concluded that to change $S A B$ and IAB, 12 sessions of NFT performed twice a week are sufficient for nonathlete people but not for athletes.

\section{Performance Tests Results}

The results obtained in the performance tests and compared between groups (Table 2) allow to infer 
that for the reaction time test there are differences; that is, both the control group (athletes) and the athletes group had initial values much higher than the nonathlete population. This first analysis makes it possible to conclude that populations are different for reaction times.

Likewise, the results for the DS performance test revealed improvements for control and athletes groups, and the OB performance test revealed improvements within the athletes group over time. There are several conclusions that can be drawn from Table 3. First of all, NFT sessions are not associated with an increase in performance tests seeing that the only group that managed to increase $S A B$ and $I A B$ over time was the nonathlete group but this group do not improve both performance tests after 12 NFT sessions. Secondly, the short-term memory test does not seem to be affected by the NFT sessions insomuch as both the control group and the athletes group have improved. Thirdly and most importantly, the reaction time is influenced by NFT sessions.

\section{Literature-supported Results}

These results are supported by the analyzed literature. The number of sessions shown sufficient is consistent with systematic reviews (Mirifar et al., 2017). The results found in DS to improve short-term memory in this study are also supported by the literature in nonathletic populations (Escolano, Aguilar, \& Minguez, 2011; Nan et al., 2012). Escolano and colleagues (2011) showed significant results in a healthy population after five consecutive training sessions (Escolano et al., 2011). On the other hand, Nan and collaborators (2012) also obtained positive results in students but only after 20 sessions (Nan et al., 2012). Regarding reaction time, robust literature exists supporting alpha wave association with that performance indicator (Klimesch, 1999).

The higher initial values of performance tests and the results obtained in reaction time in athletes follow the same line that supports that physically active people can be more efficient in more demanding executive tasks in young adults (Kamijo \& Takeda, 2010; Themanson, Pontifex, \& Hillman, 2008).

\section{Strengths, Limitations, and Considerations of the Study}

The main strength of the study is that it answers one of the major limitations pointed out by the scientific community that hypothesized whether a personalized protocol should be used for athletes other than those used in nonathletes. This study, including a nonathlete group, is able to make this contribution to the scientific community (Mirifar et al., 2017). It was also adapted the Klimesch individualized NFT (Klimesch, 1999). SAB scores were also mentioned for terms of reference and comparison. A control group was used to ensure that learning depended on NFT and not on other factors. The individualized NFT and the control group are two factors of robustness (Mirifar et al., 2017; Xiang, Hou, Liao, Liao, \& Hu, 2018).

There are limitations that should be considered:

- only marginal significant results were found in some parameters probably due to the sample size;

- a questionnaire or scale is needed to better understand both what strategies athletes are using during NFT and mood (Gruzelier, 2014);

- there were a large diversity of sports; and

- the athletes and nonathlete groups had not exactly the same protocol.

The present study should therefore be considered exploratory.

The NFT in athletes can be used as a complement to the training assuming that even 12 sessions improve the reaction time. In clinical settings, memory and reaction optimization could also have a positive impact in clinical neuropsychological tasks, improving global cognitive efficiency.

\section{Conclusions and Future Research}

The athletes showed greater improvement in reaction time than the nonathlete group and control group. In this study NFT increased the power of the bands in the nonathlete group; however, only the athletes improve performance tests after 12 NFT sessions.

This study has two important conclusions: (a) changes in SAB and IAB do not mean that there are automatically positive results in the performance tests applied and (b) 12 sessions of NFT are indeed important to notice positive changes in results at the reaction time in athletes. In other words, NFT produced a positive contribution in athletes for this study and produced a positive trend in both study groups.

Future research should replicate this protocol based on a pretest and posttest associated to the sport. Likewise, it would be necessary to compare with a three session per week protocol to verify if there are changes in $S A B$ and $I A B$ that lead to even more determining results. 


\section{Acknowledgements}

This work was supported with a grant by FCT [PestOE/EEI/LA0009/2013].

\section{Author Disclosures}

The authors declare that the research was conducted in the absence of any commercial or financial relationships that could be construed as a potential conflict of interest.

\section{References}

Angelakis, E., Stathopoulou, S., Frymiare, J. L., Green, D. L., Lubar, J. F., \& Kounios, J. (2007). EEG neurofeedback: A brief overview and an example of peak alpha frequency training for cognitive enhancement in the elderly. The Clin Neuropsychologistigist, 21(1), 110-129. https://doi.org $/ 10.1080 / 13854040600744839$

Babiloni, C., Marzano, N., Infarinato, F., lacoboni, M., Rizza, G., Aschieri, P., ... Del Percio, C. (2010). "Neural efficiency" of experts' brain during judgment of actions: A high-resolution EEG study in elite and amateur karate athletes. Behavioural Brain Research, 207(2), 466-475. https://doi.org /10.1016/j.bbr.2009.10.034

Baker, J., Côté, J., \& Deakin, J. (2005). Expertise in ultraendurance triathletes early sport involvement, training structure, and the theory of deliberate practice. Journal of Applied Sport Psychology, 17(1), 64-78. https://doi.org /10.1080/10413200590907577

Bazanova, O., \& Mernaya, E. (2008). Voluntary modification of musical performance by neurofeedback training. Annals of General Psychiatry, 7(1), S100. https://doi.org/10.1186/1744859X-7-S1-S100

Bazanova, O. M., \& Vernon, D. (2014). Interpreting EEG alpha activity. Neuroscience \& Biobehavioural Reviews, 44, 94-110. https://doi.org/10.1016/j.neubiorev.2013.05.007

Cross, E. S., Acquah, D., \& Ramsey, R. (2014). A review and critical analysis of how cognitive neuroscientific investigations using dance can contribute to sport psychology. International Review of Sport and Exercise Psychology, 7(1), 42-71. https://doi.org/10.1080/1750984X.2013.862564

Da Silva, F. L. (2013). EEG and MEG: Relevance to neuroscience. Neuron, $\quad 80(5), \quad 1112-1128 . \quad$ https://doi.org/10.1016 /j.neuron.2013.10.017

Debener, S., Makeig, S., Delorme, A., \& Engel, A. K. (2005). What is novel in the novelty oddball paradigm? Functional significance of the novelty P3 event-related potential as revealed by independent component analysis. Cognitive Brain Research, 22(3), 309-321. https://doi.org/10.1016 /j.cogbrainres.2004.09.006

Egner, T., \& Gruzelier, J. H. (2001). Learned self-regulation of EEG frequency components affects attention and event-related brain potentials in humans. NeuroReport, 12(18), 4155-4159. https://doi.org/10.1097/00001756-200112210-00058

Escolano, C., Aguilar, M., \& Minguez, J. (2011). EEG-based upper alpha neurofeedback training improves working memory performance. Paper presented at the 2011 Annual International Conference of the IEEE Engineering in Medicine and Biology Society, Boston, MA.

Gruzelier, J. H. (2014). Differential effects on mood of 12-15 (SMR) and 15-18 (beta1) $\mathrm{Hz}$ neurofeedback. International Journal of Psychophysiology, 93(1), 112-115. https://doi.org/10.1016 j.ijpsycho.2012.11.007

Guez, J., Rogel, A., Getter, N., Keha, E., Cohen, T., Amor, T., ... Gordon, S., Meiran, N., \& Todder, D. (2015). Influence of electroencephalography neurofeedback training on episodic memory: A randomized, sham-controlled, double-blind study.
Memory, 23(5), 683-694. https://doi.org/10.1080 /09658211.2014.921713

Haier, R. J., Siegel, B., Tang, C., Abel, L., \& Buchsbaum, M. S. (1992). Intelligence and changes in regional cerebral glucose metabolic rate following learning. Intelligence, 16(3-4), 415426. https://doi.org/10.1016/0160-2896(92)90018-M

Harkness, T. (2009). Psykinetics and biofeedback: Abhinav Bindra wins India's first-ever individual gold medal in Beijing olympics. Biofeedback, 37(2), 48-52. https://doi.org/10.5298/10815937-37.2.48

Hatfield, B. D., Haufler, A. J., \& Spalding, T. W. (2006). A cognitive neuroscience perspective on sport performance. In $\mathrm{E}$. Ekkekakis, \& E. Acevedo (Eds.), Psychobiology of Phshysical Activity (pp. 221-240). Champaign, IL: Human Kinetics.

Hosseini, F., \& Norouzi, E. (2017). Effect of neurofeedback training on self-talk and performance in elite and non-elite volleyball players. Medicina Dello Sport, 70(3), 344-353. https://doi.org/10.23736/S0025-7826.16.03011-8

Kamata, A., Tenenbaum, G., \& Hanin, Y. L. (2002). Individual zone of optimal functioning (IZOF): A probabilistic estimation. Journal of Sport and Exercise Psychology, 24(2), 189-208. https://doi.org/10.1123/jsep.24.2.189

Kamijo, K., \& Takeda, Y. (2010). Regular physical activity improves executive function during task switching in young adults. International Journal of Psychophysiology, 75(3), 304-311. https://doi.org/10.1016/j.ijpsycho.2010.01.002

Klimesch, W. (1999). EEG alpha and theta oscillations reflect cognitive and memory performance: A review and analysis. Brain Research Reviews, 29(2-3), 169-195. https://doi.org /10.1016/S0165-0173(98)00056-3

Landers, D. M., Petruzzello, S. J., Salazar, W., Crews, D. J., Kubitz, K. A., Gannon, T. L., \& Han, M. (1991). The influence of electrocortical biofeedback on performance in pre-elite archers. Medicine \& Science in Sports \& Exercise, 23(1), 123129. https://doi.org/10.1249/00005768-199101000-00018

Mann, C. A., Sterman, M. B., \& Kaiser, D. A. (1996). Suppression of EEG rhythmic frequencies during somato-motor and visuomotor behavior. International Journal of Psychophysiology, 23(1-2), 1-7. https://doi.org/10.1016/0167-8760(96)00036-0

Marzbani, H., Marateb, H. R., \& Mansourian, M. (2016). Neurofeedback: A comprehensive review on system design, methodology and clinical applications. Basic and Clinical Neuroscience, $7(2), \quad$ 143-158. https://doi.org/10.15412 J.BCN.03070208

Maszczyk, A., Gołaś, A., Pietraszewski, P., Kowalczyk, M., Cięszczyk, P., Kochanowicz, A., ... Zajac, A. (2018). Neurofeedback for the enhancement of dynamic balance of judokas. Biology of Sport, 35(1), 99-102. https://doi.org /10.5114/biolsport.2018.71488

Milton, J., Solodkin, A., Hluštík, P., \& Small, S. L. (2007). The mind of expert motor performance is cool and focused. Neurolmage, 35(2), 804-813. https://doi.org/10.1016 /j.neuroimage.2007.01.003

Mirifar, A., Beckmann, J., \& Ehrlenspiel, F. (2017). Neurofeedback as supplementary training for optimizing athletes' performance: A systematic review with implications for future research. Neuroscience \& Biobehavioral Reviews, 75, 419-432. https://doi.org/10.1016/j.neubiorev.2017.02.005

Nan, W., Rodrigues, J. P., Ma, J., Qu, X., Wan, F., Mak, P.-I., ... Rosa, A. (2012). Individual alpha neurofeedback training effect on short term memory. International Journal of Psychophysiology, 86(1), 83-87. https://doi.org/10.1016 j.ijpsycho.2012.07.182

Nan, W., Wan, F., Lou, C. I., Vai, M. I., \& Rosa, A. (2013). Peripheral visual performance enhancement by neurofeedback training. Applied Psychophysiology and Biofeedback, 38(4), 285-291. https://doi.org/10.1007/s10484013-9233-6

Noakes, T. D. (2012). Fatigue is a brain-derived emotion that regulates the exercise behavior to ensure the protection of 
whole body homeostasis. Frontiers in Physiology, 3, 82. https://doi.org/10.3389/fphys.2012.00082

Park, J. L., Fairweather, M. M., \& Donaldson, D. I. (2015). Making the case for mobile cognition: EEG and sports performance. Neuroscience \& Biobehavioral Reviews, 52, 117-130. https://doi.org/10.1016/j.neubiorev.2015.02.014

Pfurtscheller, G., Neuper, C., Ramoser, H., \& Müller-Gerking, J. (1999). Visually guided motor imagery activates sensorimotor areas in humans. Neuroscience Letters, 269(3), 153-156. https://doi.org/10.1016/S0304-3940(99)00452-8

Rodrigues, J. P., Migotina, D. G., \& da Rosa, A. C. (2010). EEG training platform: Improving brain-computer interaction and cognitive skills. Paper presented at the 3rd International Conference on Human System Interaction, Rzeszow, Poland. https://doi.org/10.1109/HSI.2010.5514535.

Salazar, W., Landers, D. M., Petruzzello, S. J., Han, M., Crews, D. J., \& Kubitz, K. A. (1990). Hemispheric asymmetry, cardiac response, and performance in elite archers. Research Quarterly for Exercise and Sport, 61(4), 351-359. https://doi.org/10.1080/02701367.1990.10607499

Themanson, J. R., Pontifex, M. B., \& Hillman, C. H. (2008). Fitness and action monitoring: Evidence for improved cognitive flexibility in young adults. Neuroscience, 157(2), 319-328. https://doi.org/10.1016/j.neuroscience.2008.09.014

Thompson, M., \& Thompson, L. (2015). The neurofeedback book: An introduction to basic concepts in applied psychophysiology (2nd ed.). Wheat Ridge, CO: Association for Applied Psychophysiology and Biofeedback (AAPB).

Thompson, T., Steffert, T., Ros, T., Leach, J., \& Gruzelier, J. (2008). EEG applications for sport and performance. Methods, 45(4), 279-288. https://doi.org/10.1016/j.ymeth.2008.07.006

Vernon, D. J. (2005). Can neurofeedback training enhance performance? An evaluation of the evidence with implications for future research. Applied Psychophysiology and Biofeedback, 30(4), 347. https://doi.org/10.1007/s10484-005$8421-4$
Walsh, V. (2014). Is sport the brain's biggest challenge? Current Biology, 24(18), R859-R860. https://doi.org/10.1016 /j.cub.2014.08.003

Wilson, V. E., Peper, E., \& Moss, D. (2006). "The Mind Room" in Italian soccer training: The use of biofeedback and neurofeedback for optimum performance. Biofeedback, 34(3).

World Health Organization. (2010). Global recommendations on physical activity for health. Geneva, Switzerland: World Health Organization. Retrieved from http://whqlibdoc.who.int /publications/2010/9789241599979_eng.pdf

World Medical Association. (2001). World Medical Association Declaration of Helsinki. Ethical principles for medical research involving human subjects. Bulletin of the World Health Organization, 79(4), 373

Xiang, M.-Q., Hou, X.-H., Liao, B.-G., Liao, J.-W., \& Hu, M. (2018). The effect of neurofeedback training for sport performance in athletes: A meta-analysis. Psychology of Sport and Exercise, 36, 114-122. https://doi.org/10.1016 /j.psychsport.2018.02.004

YuLeung To, E., Abbott, K., Foster, D. S., \& Helmer, D. (2016). Working memory and neurofeedback. Applied Neuropsychology: Child, 5(3), 214-222. https://doi.org /10.1080/21622965.2016.1167500

Ziółkowski, A., Graczyk, M., Strzałkowska, A., Wilczyńska, D., Włodarczyk, P., \& Zarańska, B. (2012). Neuronal, cognitive and social indicators for the control of aggressive behaviors in sport. Acta Neuropsychologica, 10(4), 537-546. https://doi.org /10.5604/17307503.1030215

Received: September 1, 2019

Accepted: February 11, 2020

Published: March 25, 2020 